УДК 62-83:621.313

\title{
АНАЛИТИЧЕСКОЕ РЕШЕНИЕ ДЛЯ ШЕСТИМАССОВОЙ ТЕРМОДИНАМИЧЕСКОЙ МОДЕЛИ АСИНХРОННОГО ДВИГАТЕЛЯ ЗАКРЫТОГО ИСПОЛНЕНИЯ
}

\author{
Ершов Михаил Сергеевич1, \\ msershov@yandex.ru
}

\section{Феоктистов Евгений Алексеевич2,} Eugene.Feoktistov@yandex.ru

\author{
1 Российский государственный университет нести и газа \\ (национальный исследовательский университет) имени И.М. Губкина, \\ Россия, 119991, г. Москва, пр. Ленинский, 65, корп. 1. \\ 2 Московский физико-технический институт (национальный исследовательский университет),
Россия, 141701, г. Долгопрудный, Институтский пер., 9.
}

\begin{abstract}
Актуальность исследования определена тем, что тепловые режимы работы асинхронных двигателей во многом определяют их надежность. В настоящее время хорошо проработаны вопросы расчета установившихся тепловых режимов двигателей. Вопросы практического расчета переходных тепловых процессов проработаны меньше и ограничены численным моделированием многомассовых схем или аналитическими решениями для моделей с несколькими массами. В данной работе предложена методика и получено аналитическое решение для типовой шестимассовой модели тепловых процессов асинхронного двигателя закрытого исполнения - Total Enclosed Fan Cooled. Полученные аналитические решения системы неоднородных линейных диффференциальных уравнений выражаются рядом, включающим постоянную составляющую и экспоненциальные члены, убывающие во времени, один из которых является доминирующим, определяющим регуляризацию процесса нагрева и охлаждения машины. Полученные аналитические решения позволяют лучше понять закономерности тепловых процессов двигателей при изменении режимов их работы и могут служить основанием для прогнозирования процессов нагрева и охлаждения в системах релейной защиты асинхронных двигателей закрытого исполнения.

Цель: разработать методику аналитических решений для многомассовых термодинамических моделей электрических машин, получить аналитическое решение для типовой шестимассовой системы асинхронного двигателя закрытого исполнения и выполнить анализ полученных результатов.

объекты: асинхронные двигатели в закрытом исполнении.

Методы: аналитическое решение системы неоднородных диффреренциальных уравнений.

Результаты. Разработана методика аналитических решений для многомассовых термодинамических моделей электрических машин, получено аналитическое решение для шестимассовой системы асинхронного двигателя закрытого исполнения и выполнен анализ полученных результатов, установлены закономерности регуляризации процессов нагрева объекта исследования.
\end{abstract}

\section{Ключевые слова:}

Асинхронный двигатель, закрытое исполнение, термодинамическая модель,

система неоднородных дифрференциальных уравнений, аналитическое решение, анализ.

\section{Введение}

Асинхронные двигатели мощностью до 100 кВт обычно выполняются в закрытом обдуваемом исполнении, в котором применена искусственная циркуляция охлаждающего воздуха с помощью вентиляторов $[1,2]$ - Total Enclosed Fan Cooled (TEFC) [3, 4].

Такие машины получили широкое распространение во всех отраслях промышленности. В нефтяной и газовой промышленности на долю двигателей в закрытом обдуваемом исполнении приходится свыше $50 \%$ численного состава двигателей. Асинхронные двигатели серий $\mathrm{AO}, 4 \mathrm{~A}, \mathrm{AИP}, \mathrm{RA}, 5 \mathrm{~A}, 6 \mathrm{~A}$ являются основными в приводах систем водоподготовки и водоснабжения, вентиляции, механизированной добычи нефти станками-качалками и в других производствах добычи и переработки нефти и газа, где не требуется применения взрывозащищенного оборудования. Надежность двигателей во многом определяется тепловыми режимами их работы, нагревом обмоток двигателей. Для теплового расчета асинхронных двига- телей обычно применяется метод эквивалентных тепловых схем [1-4], которые рассчитываются относительно характерных режимов [5] с определением стационарных температур основных элементов многомассовых типовых схем. Более сложной является задача расчета переходных тепловых процессов, связанных с изменением режимов работы двигателей, требующая построения термодинамических моделей. Обычно такие задачи решаются только в численном виде. Аналитические решения ограничены случаями одно-, двух- и трехмассовых термодинамических моделей [6-8]. В то же время аналитические решения для термодинамических моделей позволяют увидеть и лучше понять особенности и закономерности тепловых процессов электрических машин при изменении режимов их работы, на которых строятся алгоритмы работы цифровых защит двигателей [9-12].

\section{Постановка и исходные данные задачи}

Задачей данной статьи является разработка методики аналитических решений для многомассовых 
термодинамических моделей электрических машин, получение аналитического решения для шестимассовой системы асинхронного двигателя закрытого исполнения, выполнение анализа и выявление закономерностей нагрева двигателя при изменении режима его работы. Под аналитическим решением понимается получение зависимостей температуры элементов двигателя во времени, при этом параметры (коэффициенты) зависимостей рассчитываются через параметры тепловой схемы машины. Для выполнения исследований была разработана математическая модель короткозамкнутого асинхронного двигателя (АД) закрытого обдуваемого исполнения. Модель построена на базе типовой шестимассовой тепловой схемы $[1,2]$, приведенной на рис. 1.

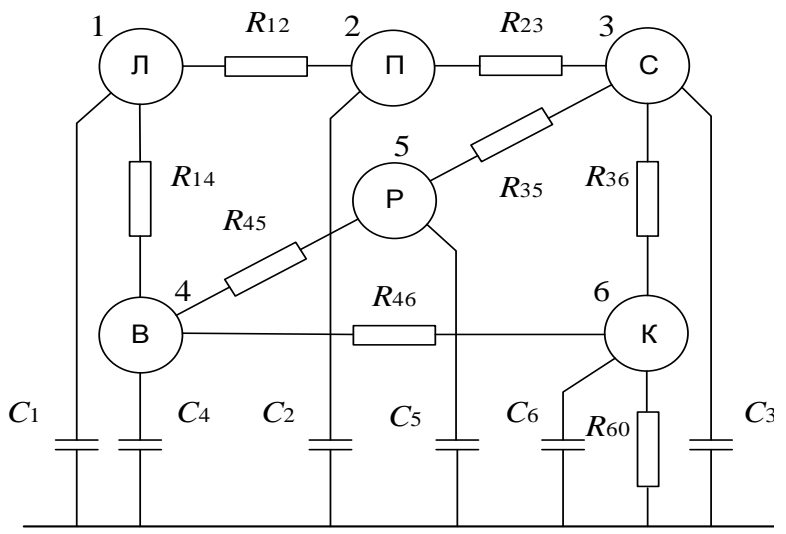

Рис. 1. Тепловая схема АД закрытого исполнения: 1. Ллобовая часть статорной обмотки; 2. П-пазовая часть статорной обмотки; 3. C-cтатор; 4. $B-$ воздух; 5. P-poтор; 6. K-корпус; $R_{i k}$ соответствующие тепловые сопротивления; $C_{i}-$ теплоемкости элементов (узлов) схемы

Fig. 1. Thermal diagram of the closed-circuit IM: 1. Л-the frontal part of the stator winding; $2 . \Pi$-the grooved part of the stator winding; 3 . $C$ - the stator; $4 . B-$ air; 5. $P$-the rotor; $6 . K$ - the housing; $R_{i k}-$ the corresponding thermal resistances; $C_{i}$ - the heat capacity of the elements (nodes) of the circuit

Математическая модель нестационарных тепловых процессов двигателя представляет собой систему дифференциальных уравнений:

$$
\begin{gathered}
\frac{d \tau_{1}}{d t}=\frac{\left(-\Lambda_{1} \tau_{1}+\Lambda_{12} \tau_{2}+\Lambda_{14} \tau_{4}+\Delta P_{1}\right)}{C_{1}} ; \\
\frac{d \tau_{2}}{d t}=\frac{\left(\Lambda_{12} \tau_{1}-\Lambda_{2} \tau_{2}+\Lambda_{23} \tau_{3}+\Delta P_{2}\right)}{C_{2}} ; \\
\frac{d \tau_{3}}{d t}=\frac{\left(\Lambda_{23} \tau_{2}-\Lambda_{3} \tau_{3}+\Lambda_{35} \tau_{5}+\Lambda_{36} \tau_{6}+\Delta P_{3}\right)}{C_{3}} ; \\
\frac{d \tau_{4}}{d t}=\frac{\left(\Lambda_{14} \tau_{1}-\Lambda_{4} \tau_{4}+\Lambda_{45} \tau_{5}+\Lambda_{46} \tau_{6}+\Delta P_{4}\right)}{C_{4}} ; \\
\frac{d \tau_{5}}{d t}=\frac{\left(\Lambda_{35} \tau_{3}+\Lambda_{45} \tau_{4}-\Lambda_{5} \tau_{5}+\Delta P_{5}\right)}{C_{5}} ; \\
\frac{d \tau_{6}}{d t}=\frac{\left(\Lambda_{36} \tau_{3}+\Lambda_{46} \tau_{4}-\Lambda_{6} \tau_{6}+\Delta P_{6}\right)}{C_{6}} ;
\end{gathered}
$$

где $\tau_{i}$ - значение превышения температуры $i$-го узла над температурой окружающей среды; $\Lambda_{i k}-$ взаимная тепловая проводимость между $i$-м и $k$-м узлами схемы; $\Lambda_{i}$ - узловая тепловая проводимость $i$-го узла; $C_{i}-$ теплоемкость $i$-го узла; $\Delta P_{i}$ - потери мощности в $i$-м узле.

Взаимные тепловые проводимости определяются как величины, обратные соответствующим тепловым сопротивлениям. Значения узловых тепловых проводимостей определяются как сумма проводимостей всех связанных с данным узлом ветвей. Влияние температуры на теплоемкости и теплопроводности в режимах реальных значений температур незначительно, на первых этапах исследования им можно пренебречь [4, 13]. В этом случае система дифференциальных уравнений (1) является линейной.

В качестве объекта исследования переходных процессов нагревания и охлаждения выбран асинхронный короткозамкнутый двигатель закрытого обдуваемого исполнения типа 4А112М4 мощностью 5,5 кВт; напряжение $U_{\text {ном }}=380 \mathrm{~B}$; КПД $\eta_{\text {ном }}=0,85$; скольжение $s_{\text {ном }}=0,036$. Данный тип двигателей относится по степени защиты от воздействия среды к категории IP44 и по типу охлаждения IC0141.

Основные исходные данные для расчетов взяты из работы [1] и составили:

- тепловые сопротивления схемы равны следующим значениям:

$$
R_{12}=0,443 ; R_{14}=0,1358 ; R_{23}=0,0432 ; R_{35}=0,1562 ;
$$

$R_{36}=0,0286 ; R_{45}=0,1643 ; R_{46}=0,0711 ; R_{60}=0,0296, \frac{\mathrm{K}}{\mathrm{BT}}$,

- вектор теплоемкостей элементов схемы имеет вид

$$
C_{i}=(1611 ; 1383 ; 7885 ; 4,4 ; 8256 ; 5034), \frac{\text { Дж }}{\mathrm{K}} .
$$

При определении теплоемкости воздуха его расход принят равным $0,0785 \mathrm{~m}^{3} / \mathrm{c}$.

Электрические потери в статорной обмотке определяются по формуле

$$
\Delta P_{\text {эл. } 1}=3 I_{1}^{2} r_{1},
$$

где $I_{1}$ - ток статорной обмотки; $r_{1}$ - сопротивление статорной обмотки.

Потери в лобовой и пазовой части статорной обмотки распределены пропорционально длине соответственно лобовой и пазовой частей статорной обмотки. Мощность потерь в стали статора определяется как сумма мощности основных потерь в стали и половины добавочных потерь $\Delta P_{3}=\Delta P_{\text {мг }}+0,5 \Delta P_{\text {доб. }}$ Потери в четвертом и шестом элементах приняты равными четвертой части механических потерь $\Delta P_{4}=\Delta P_{6}=0,25 \Delta P_{\text {мех }}$. Потери в пятом элементе определены как сумма электрических потерь в обмотке ротора и половина добавочных потерь $\Delta P_{5}=\Delta P_{\text {эл.2 }}+0,5 \Delta P_{\text {доб. }}$ Значение магнитных потерь в статоре, механических и добавочных потерь принято равным соответственно 25,10 и $5 \%$ от величины общих потерь, определяемых по значениям мощности на валу двигателя и КПД $\Delta P=P(1-\eta) / \eta$. Мощность электрических потерь в роторе определена произведением скольжения на электромагнитную мощность, в свою очередь опреде- 
ляемую как разность общих потерь, электрических и магнитных потерь в статоре

$$
\Delta P_{\text {эл.2 }}=s P_{\text {эм }}=s\left(\Delta P-\Delta P_{\text {эл.1 }}-\Delta P_{\text {мг }}\right)[5] .
$$

Вектор потерь мощностей в элементах схемы для номинального режима имеет вид

$$
\Delta P_{i}=(291 ; 225 ; 289 ; 10 ; 278 ; 10), \text { Вт. }
$$

\section{Методика аналитического решения для термодинамической модели}

Если подставить в систему уравнений (1) численные значения, то получим неоднородную систему линейных дифференциальных уравнений, которую удобно представить в матричном виде

$$
\dot{\boldsymbol{\tau}}(t)=\mathbf{A} \cdot \boldsymbol{\tau}(t)+\mathbf{b},
$$

где $\dot{\boldsymbol{\tau}}(t)$ - вектор-столбец производных превышения температуры (6×1); А - матрица коэффициентов (6×6); $\tau(t)$ - вектор-столбец превышения температур; b вектор-столбец свободных членов $(6 \times 1)$.

Тогда соответствующая системе (2) однородная система линейных дифференциальных уравнений запишется в виде

$$
\dot{\boldsymbol{\tau}}(t)=\mathbf{A} \cdot \boldsymbol{\tau}(t) .
$$

Известно, что общее решение системы (2) есть сумма общего решения системы (3) и любого частного решения системы (2). В нашем случае матрица А и вектор-столбец $\mathbf{b}$ имеют следующий вид

$$
\begin{gathered}
\mathbf{A}=\left(\begin{array}{cccccc}
-0,02315 & 0,01401 & 0 & 0,00914 & 0 & 0 \\
0,01632 & -0,03306 & 0,01673 & 0 & 0 & 0 \\
0 & 0,00293 & -0,00818 & 0 & 0,00081 & 0,00443 \\
0,33471 & 0 & 0 & -0,79269 & 0,13833 & 0,31965 \\
0 & 0 & 0,00078 & 0,00074 & -0,00151 & 0 \\
0 & 0 & 0,00694 & 0,00279 & 0 & -0,01645
\end{array}\right) ; \\
\mathbf{b}=\left(\begin{array}{c}
0,18063 \\
0,16269 \\
0,03665 \\
0,02272 \\
0,03367 \\
0,00199
\end{array}\right) .
\end{gathered}
$$

На первом шаге найдем общее решение однородной системы (3). Для этого необходимо найти все собственные числа $\lambda_{i}$ и соответствующие им собственные векторы $\mathbf{h}_{i}$ матрицы А. Из последних в свою очередь составим так называемую фундаментальную матрицу решений системы (3). В дальнейшем обозначим ее буквой Н. При обработке в Matlab [14] собственные числа удобно хранить в виде следующего вектор-столбца

$$
\lambda=\left(\begin{array}{l}
\lambda_{1} \\
\lambda_{2} \\
\lambda_{3} \\
\lambda_{4} \\
\lambda_{5} \\
\lambda_{6}
\end{array}\right)=\left(\begin{array}{l}
-0,79792 \\
-0,04388 \\
-0,00070 \\
-0,00290 \\
-0,01187 \\
-0,01778
\end{array}\right) .
$$

Все собственные числа являются вещественными и различными, что говорит о том, что общее решение однородной системы уравнений будет иметь следующий вид

$$
\boldsymbol{\tau}_{i . o d}=\left(\begin{array}{c}
\tau_{1} \\
\ldots \\
\tau_{6}
\end{array}\right)=\sum_{i=1}^{6} K_{i} \cdot e^{\lambda_{i} \cdot t} \cdot \mathbf{h}_{i},
$$

где $K_{i}$ - некоторые вещественные константы, определяемые ниже.

Определив с помощью стандартных процедур Matlab собственные векторы, составим фундаментальную матрицу решений

$$
\mathbf{H}=\left(\begin{array}{cccccc}
0,01180 & -0,48503 & 0,37558 & -0,46591 & 0,80421 & 0,13018 \\
-0,00025 & 0,84731 & 0,36677 & -0,51256 & 0,45851 & -0,20864 \\
-0,00002 & -0,07466 & 0,34277 & -0,46920 & -0,20390 & -0,31741 \\
-0,99992 & -0,19918 & 0,36020 & -0,24656 & 0,28944 & 0,39631 \\
0,00093 & 0,00483 & 0,65644 & 0,39291 & -0,00533 & -0,00283 \\
0,00358 & 0,03920 & 0,21509 & -0,29136 & -0,13277 & 0,82566
\end{array}\right)
$$

Напомним, что в ней $i$-й столбец является $i$-м собственным вектор-столбцом $\mathbf{h}_{i}$. Таким образом, общее решение однородной системы (3) получено.

На втором шаге найдем частные решения неоднородной системы (2). В силу того, что подойдет любое частное решение, будем искать его в виде набора постоянных функций

$$
\boldsymbol{\tau}_{i . n o d}=\left(\begin{array}{c}
\tau_{1} \\
\cdots \\
\tau_{6}
\end{array}\right)_{\text {nod }}=\boldsymbol{\alpha}=\left(\begin{array}{c}
\alpha_{1} \\
\ldots \\
\alpha_{6}
\end{array}\right) .
$$

Значение вектора $\boldsymbol{\alpha}$ определим, формально, подставив его в систему уравнений (2). Полагая при этом, что в установившемся режиме, то есть при $t \rightarrow \infty$, справедливо $\dot{\boldsymbol{\tau}}(t)=0$, получим следующую систему уравнений

$$
\mathbf{A} \cdot \boldsymbol{\alpha}=-\mathbf{b} .
$$

Численные значения матрицы $\mathbf{A}$ и вектора $\mathbf{b}$ приведены выше. Решение системы уравнений (5) относительно вектора $\boldsymbol{\alpha}$, осуществленное в Matlab, имеет вид

$$
\boldsymbol{\alpha}=\left(\begin{array}{l}
\alpha_{1} \\
\alpha_{2} \\
\alpha_{3} \\
\alpha_{4} \\
\alpha_{5} \\
\alpha_{6}
\end{array}\right)=\left(\begin{array}{l}
70,596312 \\
66,978656 \\
53,730829 \\
56,382307 \\
77,283585 \\
32,382400
\end{array}\right) .
$$

Таким образом, получили решение исходной системы, которое с учетом выражения (4) примет вид

$$
\boldsymbol{\tau}_{i}=\boldsymbol{\tau}_{i . o d}+\boldsymbol{\tau}_{i . n o d}=\sum_{i=1}^{6} K_{i} \cdot e^{\lambda_{i} \cdot t} \cdot \mathbf{h}_{i}+\left(\begin{array}{c}
\alpha_{1} \\
\ldots \\
\alpha_{6}
\end{array}\right) .
$$

Значения вещественных констант $K_{i}$ в уравнении (6) однозначно определяются начальными условиями, принятыми из следующих физических соображений: 
1) работа двигателя начинается из холодного состояния, то есть $\tau(0)=0 ; 2)$ в начальный момент времени температура не меняется скачком, то есть $\dot{\boldsymbol{\tau}}(0)=\dot{\boldsymbol{\tau}}(-0)=0$. Подставляя эти условия в формулу (6), получим систему линейных алгебраических уравнений

$$
\mathbf{H} \cdot \mathbf{K}=-\boldsymbol{\alpha},
$$

где $H=\left(h_{1}\left|h_{2}\right| h_{3}\left|h_{4}\right| h_{5} \mid h_{6}\right)$ - фундаментальная матрица решений; K - вектор-столбец вещественных констант.

Решая систему уравнений (7) относительно $\mathbf{K}$, получим

$$
\mathbf{K}=\left(\begin{array}{l}
K_{1} \\
K_{2} \\
K_{3} \\
K_{4} \\
K_{5} \\
K_{6}
\end{array}\right)=\left(\begin{array}{c}
-0,07543 \\
-0,47099 \\
-131,75953 \\
23,27201 \\
-13,24502 \\
1,20965
\end{array}\right) .
$$

Выполнив перемножение вектора $\mathbf{K}$ и матрицы $\mathbf{H}$ на основании выражения (6), получим аналитическое решение исходной системы дифференциальных уравнений (1) для принятых начальных условий в виде

$$
\begin{aligned}
& \left(\begin{array}{c}
\tau_{1} \\
\tau_{2} \\
\tau_{3} \\
\tau_{4} \\
\tau_{5} \\
\tau_{6}
\end{array}\right)=e^{-0,79792 \cdot t} \cdot\left(\begin{array}{c}
-0,000890 \\
0,000019 \\
0,000002 \\
0,075420 \\
-0,000070 \\
-0,000270
\end{array}\right)+ \\
& +e^{-0,04388 \cdot t} \cdot\left(\begin{array}{c}
0,228445 \\
-0,399078 \\
0,035167 \\
0,093812 \\
-0,002276 \\
-0,018462
\end{array}\right)+e^{-0,00070 \cdot t} \cdot\left(\begin{array}{c}
-49,486777 \\
-48,325970 \\
-45,163586 \\
-47,459274 \\
-86,492311 \\
-28,340459
\end{array}\right)+ \\
& +e^{-0,00290 \cdot t} \cdot\left(\begin{array}{c}
-10,842774 \\
-11,985086 \\
-10,986716 \\
-5,827311 \\
8,836382 \\
-6,802409
\end{array}\right)+e^{-0,01187 \cdot t} \cdot\left(\begin{array}{c}
-10,651789 \\
-6,073007 \\
2,700687 \\
-3,833686 \\
0,070643 \\
1,758576
\end{array}\right)+ \\
& +e^{-0,01778 \cdot t} \cdot\left(\begin{array}{c}
0,157472 \\
-0,252377 \\
-0,383956 \\
0,479394 \\
-0,003423 \\
0,998760
\end{array}\right)+\left(\begin{array}{c}
70,596312 \\
66,978656 \\
53,730829 \\
56,382307 \\
77,283585 \\
32,382400
\end{array}\right) \text {. }
\end{aligned}
$$

Таким образом, изменение температуры каждой массы системы во времени можно представить как линейную комбинацию шести экспонент и постоянной составляющей, соответствующей установившемуся значению температуры элемента системы по завершению теплового переходного процесса. Напри- мер, нагрев первого элемента определяется следующей суммой экспонент и постоянной составляющей

$$
\begin{gathered}
\tau_{1}(t)=-0,000890 \cdot e^{-0,79792 \cdot t}+ \\
+0,228445 \cdot e^{-0,04388 \cdot t}-49,486700 \cdot e^{-0,00070 \cdot t}- \\
-10,842774 \cdot e^{-0,00290 \cdot t}-10,651789 \cdot e^{-0,01187 \cdot t}+ \\
+0,157472 \cdot e^{-0,01778 \cdot t}+70,596312 .
\end{gathered}
$$

Согласно (8) нагрев остальных элементов определиться следующими выражениями

$$
\begin{gathered}
\tau_{2}(t)=0,000019 \cdot e^{-0,79792 \cdot t}- \\
-0,399078 \cdot e^{-0,04388 \cdot t}-48,325970 \cdot e^{-0,00070 \cdot t}- \\
-11,985086 \cdot e^{-0,00290 \cdot t}-6,073007 \cdot e^{-0,01187 \cdot t}- \\
-0,252377 \cdot e^{-0,01778 \cdot t}+66,978656 ; \\
\tau_{3}(t)=0,000002 \cdot e^{-0,79792 \cdot t}+ \\
+0,035167 \cdot e^{-0,04388 \cdot t}-45,163586 \cdot e^{-0,00070 \cdot t}- \\
-10,986716 \cdot e^{-0,00290 \cdot t}+2,700687 \cdot e^{-0,01187 \cdot t}- \\
-0,383956 \cdot e^{-0,01778 \cdot t}+53,730829 ; \\
\tau_{4}(t)=0,075420 \cdot e^{-0,79792 \cdot t}+ \\
+0,093812 \cdot e^{-0,04388 \cdot t}-47,459274 \cdot e^{-0,00070 \cdot t}- \\
-5,827311 \cdot e^{-0,00290 \cdot t}-3,833686 \cdot e^{-0,01187 \cdot t}+ \\
+0,479394 \cdot e^{-0,01778 \cdot t}+56,382307 ; \\
\tau_{5}(t)=-0,000070 \cdot e^{-0,79792 \cdot t}- \\
-0,002276 \cdot e^{-0,04388 \cdot t}-86,492311 \cdot e^{-0,00070 \cdot t}+ \\
+8,836382 \cdot e^{-0,00290 \cdot t}+0,070643 \cdot e^{-0,01187 \cdot t}- \\
-0,003423 \cdot e^{-0,01778 \cdot t}+77,283585 ; \\
\tau_{6}(t)=-0,000270 \cdot e^{-0,79792 \cdot t}- \\
-0,018462 \cdot e^{-0,04388 \cdot t}-28,340459 \cdot e^{-0,00070 \cdot t}- \\
-6,802409 \cdot e^{-0,00290 \cdot t}+1,758576 \cdot e^{-0,01187 \cdot t}+ \\
+0,998760 \cdot e^{-0,01778 \cdot t}+32,382400 .
\end{gathered}
$$

\section{Анализ результатов}

На рис. 2 приведены графики температур элементов системы при изменении мощности потерь от нулевых значений до значений потерь мощности в номинальном режиме.

На рис. 3, 4 приведены графики основных составляющих и результирующие кривые нагрева лобовой части обмотки статора и ротора. На рисунках пронумерованы только две первых экспоненциальных составляющих с наименьшими показателями степеней экспоненциальных составляющих (1. $\lambda_{3}=-0,0007$;

(8) 2. $\left.\lambda_{4}=-0,0029\right)$, поскольку остальные составляющие малы и практически сливаются с осью абсцисс.

Полученные аналитические решения (9) соответствуют теореме Г.М. Кондартьева [15], согласно которой процесс нагрева системы из однородных изотропных тел (с одинаковой во всех направлениях теплопроводностью) выражается рядом, включающим постоянную составляющую и экспоненциальные члены, абсолютные значения которых убывают во времени. Постоянный член и коэффициенты - сомножители экспоненциальных функций - конечны и не зависят от времени. 


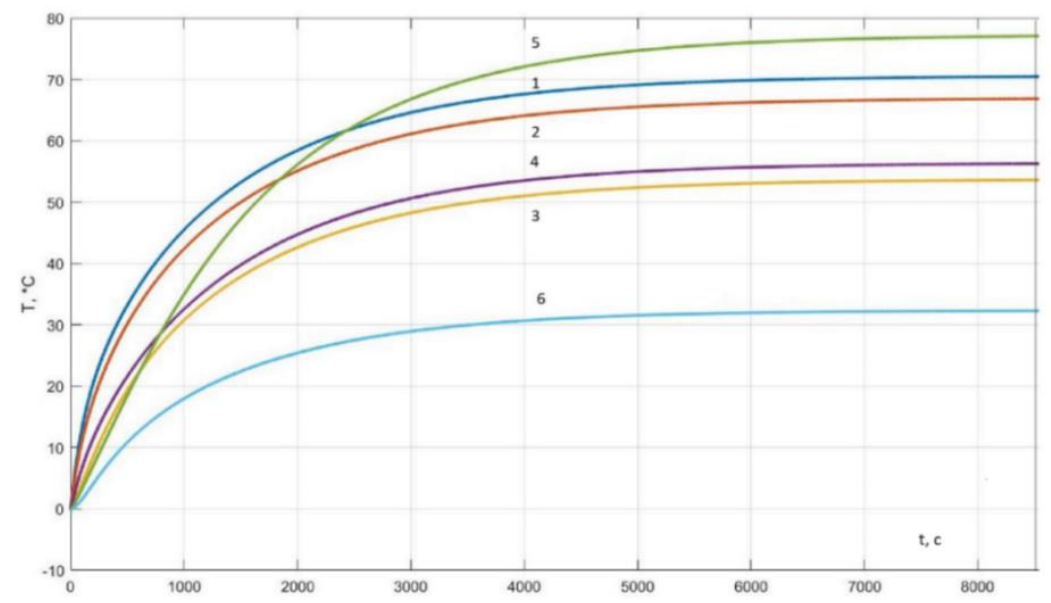

Рис. 2. Графики превышения температуры элементов системы. Номера кривых соответствуют номерам элементов тепловой схемы двигателя

Fig. 2. Graphs of the excess temperature of the system elements. The numbers of the curves correspond to the numbers of the elements of the thermal circuit of the engine

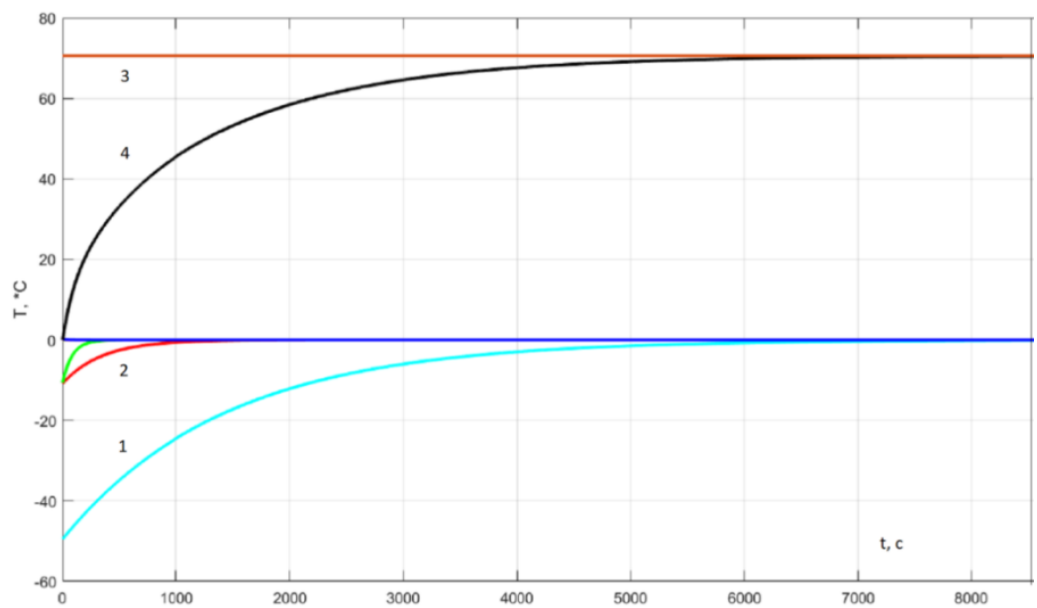

Рис. 3. Основные составляющие и результирующий график нагрева лобовой части обмотки статора: 1 - третья экспоненциальная составляющая; 2 - четвертая экспоненциальная составляющая; 3 - постоянная составляющая; 4 - результирующий график нагрева

Fig. 3. Main components and the resulting heating graph of the frontal part of the stator winding: 1 - the third exponential component; 2 - the fourth exponential component; 3 - the constant component; 4 - the resulting heating graph

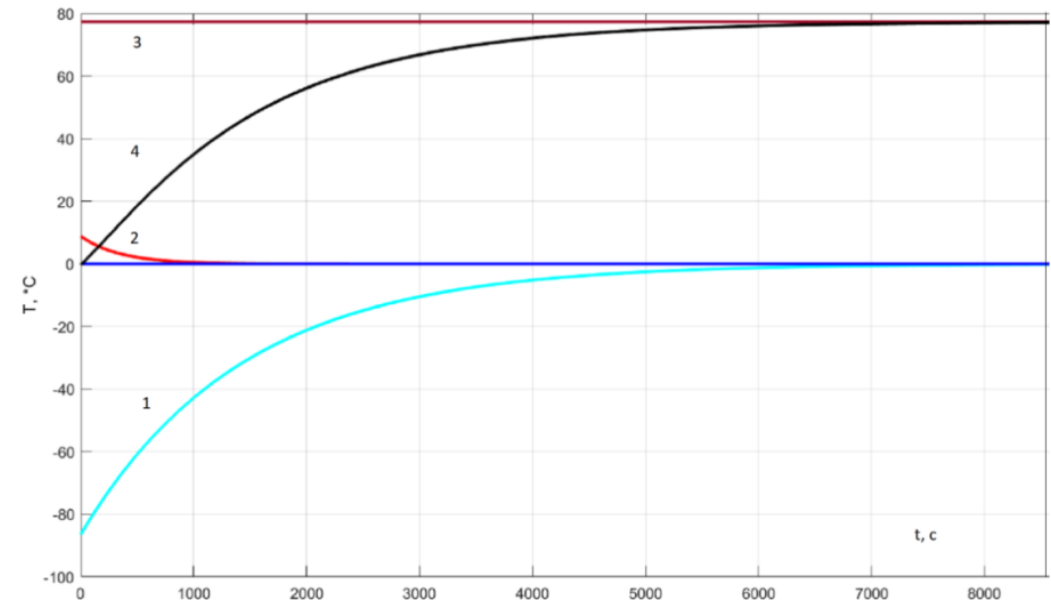

Рис. 4. Основные составляющие и результирующий график нагрева ротора: 1 - третья экспоненциальная составляющая; 2 - четвертая экспоненциальная составляющая; 3 - постоянная составляющая; 4 - результирующий график нагрева

Fig. 4. Main components and the resulting heating graph of the rotor: 1 - the third exponential component; 2 - the fourth exponential component; 3 - the constant component; 4 - the resulting heating graph 
При этом среди экспоненциальных слагаемых доминирующим является один основной член с максимальной постоянной времени, определяемой отношением $T_{\max }=1 / \min \left\{\lambda_{i}\right\}, \forall i$. Здесь $\lambda_{i}-$ показатель степени экспонент, называемый темпом теплового процесса $[15,16]$. В рассматриваемом примере $T_{\max }=1 / 0,0007=1429$ с. Остальные экспоненциальные члены ряда скоро становятся пренебрежимо малыми по сравнению с основным [15], то есть через какое-то время, в качестве которого можно взять утроенное значение второй по величине постоянной времени из слагаемых экспонент, изменение температуры будет следовать простому экспоненциальному закону.

Тепловой режим, подчиняющийся экспоненциальному закону, называется регулярным (упорядоченным) режимом. В рассматриваемом примере можно считать, что процесс нагрева становиться регулярным через время $T_{\text {per }}=3 \cdot(1 / 0,0029)=1034$ с. Это значение составляет около $70 \%$ от значения постоянной времени основной экспоненциальной слагаемой. С учетом значений коэффициентов - сомножителей экспонент, время регуляризации будет еще меньше. Регуляризация тепловых процессов позволяет проще решать практические задачи термометрии, калориметрии и прогнозирования процессов нагрева и охлаждения электрических машин [17-20].

Так, после регуляризации теплового процесса через время $t>T_{\text {рег }}$ по результатам трех измерений температуры можно определить постоянную времени $T_{\mathrm{H}}=T_{\max }[21]$. Причем из полученных решений (9) следует, что измерения можно проводить на любом элементе системы, в том числе измеряя температуру на наиболее доступном для измерения температуры элементе - корпусе двигателя

$$
T_{\text {н }}=\frac{\Delta t}{\ln \left(\frac{\tau_{2}-\tau_{1}}{\tau_{3}-\tau_{2}}\right)},
$$

где $\Delta t-$ интервал времени между измерениями; $\tau_{1}, \tau_{2}$ $\tau_{3}$ - измеренные на трех моментах времени значения превышения температуры над температурой окружающей среды.

На рис. 5 приведены значения ошибок при определении постоянной времени нагрева электродвигателя в зависимости от времени, температура при котором принимается за начальную точку отсчета $\tau_{1}$, и места измерения температуры, в качестве которого выбраны лобовая часть обмотки статора и корпус двигателя. Интервал времени между измерениями принят равным $\Delta t=500$ с. Уменьшение времени начала отсчета температуры $\tau_{1}$ до 1000 с приведет к увеличению отрицательного значения ошибки определения постоянной времени $T_{\mathrm{H}}$ до минус $6 \%$. То есть с вполне приемлемой точностью можно определить по-

\section{СПИСОК ЛИТЕРАТУРЫ}

1. Сипайлов Г.А., Санников Д.И., Жадан В.А. Тепловые, гидравлические и аэродинамические расчеты в электрических машинах. - М.: Высшая школа, 1989. - 239 с.

2. Жадан В.А., Говязова С.В. Тепловой расчет электрических машин закрытого исполнения с естественным охлаждением и стоянную времени нагревания двигателя по формуле (10) по нескольким измерениям температуры после регуляризации процесса нагрева двигателя

Вместе с тем именно при изменении режимов работы, в частности, при изменении нагрузки, температура обмотки статора, а это основная величина, характеризующая тепловое состояние двигателя, изменяется с темпом, который превосходит темп изменения экспоненты с наибольшей постоянной. И алгоритмы работы цифровой защиты должны это учитывать. При наличии аналитического решения в распоряжении разработчиков защит оказывается информация и о меньших постоянных модели, определяющих темп нагрева обмотки статора на начальном этапе.

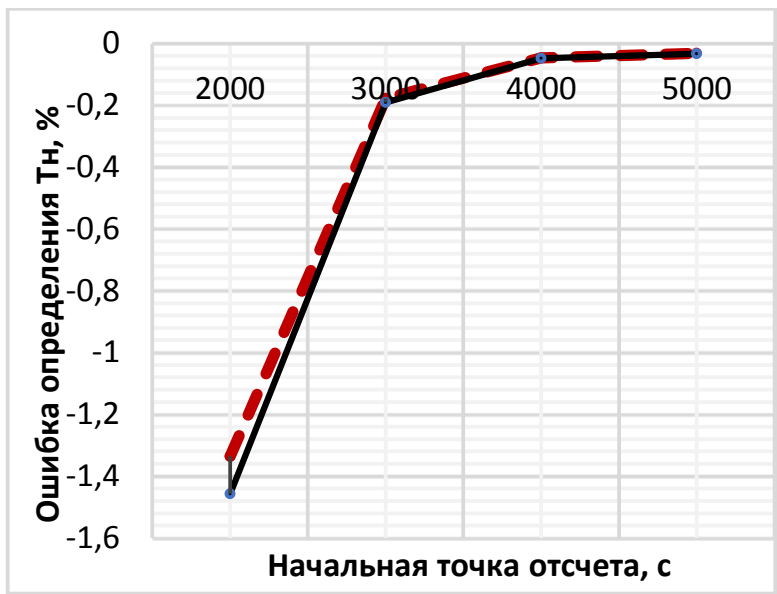

Рис. 5. Зависимость ошибки экспериментального определения постоянной времени асинхронного двигателя 4A112M4 от времени начала измерений и от элемента системы, на котором проводятся измерения: на лобовой части статорной обмотки (пунктирная линия); на корпусе двигателя (сплошная линия)

Fig. 5. Dependence of the error of the experimental determination of the asynchronous motor 4A112M4 time constant on the time of the beginning of measurements and on the system element on which measurements are carried out: on the frontal part of the stator winding (dotted line); on the motor housing (solid line)

\section{Заключение}

Разработана методика аналитических решений для многомассовых термодинамических моделей электрических машин. Получено аналитическое решение для шестимассовой системы асинхронного двигателя закрытого исполнения, и выполнен анализ полученных результатов. Установлены закономерности регуляризации процессов нагрева объекта исследования, даны рекомендации по экспериментальному определению постоянной времени асинхронного двигателя.

оребренным корпусом // Известия Томского политехнического университета. - 2005. - Т. 308. - № 7. - С. 174-178.

3. Boglietti A., Cavagnino A., Staton D. Thermal analysis of TEFC induction motors // IEEE IAS Annual Meeting. - Salt Lake City: UK, 2003. - P. 849-856. 
4. Boglietti A., Cavagnino A., Staton D.A. TEFC Induction motors thermal models: a parameter sensitivity analysis // IEEE Transactions on Industry Applications. - 2005. - V. 41. - № 3. P. 756-763.

5. German-Galkin S., Sakharov V., Tarnapowicz D. Energy characteristics of asynchronous electric drive // Management Systems in Production Engineering. - 2019. - V. 27. - № 1. P. 51-54. DOI: 10.1515/mspe-2019-0009.

6. Метельков В.П. О расчете параметров двухмассовой термодинамической модели асинхронного двигателя // Вестник Южно-Уральского государственного университета. Серия «Энергетика». - 2016. - Т. 16. - № 1. - С. 58-65. DOI: $10.14529 /$ power 160109

7. Анучин А.С., Федорова К.Г. Двухмассовая тепловая модель асинхронного двигателя // Электротехника. - 2014. - № 2 . C. 21-25.

8. Оценка теплового состояния электродвигателей переменного тока компрессорных станций магистральных газопроводов / А.М. Зюзев, О.В. Крюков, В.П. Метельков, С.Г. Михальченко // Известия Томского политехнического университета. Инжиниринг георесурсов. - 2020. - Т. 332. - № 1. - С. 88-96.

9. Математические модели нагрева и охлаждения для микропроцессорного реле тепловой защиты / Г.А. Бугаев, Е.Ю. Леонтьев, Д.В. Ерохин, Д.В. Павлова // Электротехника. - 2001. № 2. - C. 51-54.

10. Тепловая модель асинхронного двигателя для целей релейной защиты / А.В. Булычев, Е.Ю. Ерохин, Н.Д. Поздеев, О.А. Филичев // Электротехника. - 2011. - № 1. - С. 26-30.

11. Thermal modelling of an alternator for use in a prediction system / J. Graham, R. Dixon, K. Gregory, J. Pearson // Proc. of 2012 UKACC International Conference on Control (CONTROL 2012). Cardiff: UK, 2012. - P. 455-460. DOI: 10.1109/CONTROL.2012. 6334673.

12. Fundamentals of a motor thermal model and its applications in motor protection / B. Venkataraman, B. Godsey, W. Premerlani, E. Shulman, M. Thakur, R. Midence // Protective Relay Engineers, 58th Annual Conference. - USA, 2005. - P. 11-28. DOI 10.1109/CPRE.2005.1430428.

13. Modeling of thermal process in the energy system «electrical network - asynchronous motor» / V. Kuznetsov, M. Tryputen, V. Tytiuk, Zh. Rozhnenko, S. Levchenko, V. Kuznetsov // E3S
Web of Conferences ICSF. - 2021. - P. 1-6. URL: https://doi.org/10.1051/e3sconf/202128005003 (дата обращения 15.11.2021).

14. Roland P. MATLAB for electrical and computer engineering students and professional with Simulink. - Edison: SciTech Publ., NJ. University of Illinois at Chicago, 2013. - 662 p.

15. Кондратьев Г.М. Регулярный тепловой режим. - М.: Государственное изд-во технико-теоретической литературы, 1954. $408 \mathrm{c}$.

16. Hanappier N., Charkaluk E., Triantafyllidis N. A coupled electromagnetic-thermomechanical approach for the modeling of electric motors // Journal of the Mechanics and Physics of Solids. - 2021. - V. 149. - Article ID 104315. - P. 1-31. DOI: 10.1016/j.jmps.2021.104315.

17. Zocholl S. Optimizing motor thermal models // Proc. Industrial \& Commercial Power Systems Technical Conference, 2007. ICPS 2007. - Edmonton, Alberta, Canada, 2007. - P. 33-41.

18. Borodin M.Yu., Borodin E.M., Metelkov V.P. Application of integral equations for analysis of electrodynamic and thermal processes in electric drive // Proc. 2016 2nd International Conference on Industrial Engineering, Applications and Manufacturing (ICIEAM 2016). - Chelyabinsk, Russia, 2016. 7911533. - P. 1-4

19. Хомченко Д.Н., Ковалев Г.В. Исследование влияния повышенной плотности тока в щетках на процесс нагрева тягового двигателя электровоза // Вестник РГУПС. - 2019. - № 1. C. $46-52$.

20. Staton D., Boglietti A., Cavagnino A. Solving the more difficult aspects of electric motor thermal analysis in small and medium size industrial induction motors // IEEE Transaction on Energy Conversion. - 2005. - V. 20. - № 3. - P. 620-628.

21. Коренчиак Д., Себок М., Гуттен М. Тепловое измерение и его применение для диагностики масляных трансформаторов распределительных сетей // Энергетика. Известия высших учебных заведений и энергетических объединений СНГ - 2019. T. 62. - № 6. - C. 583-594. URL: https://doi.org/10.21122/10297448-2019-62-6-583-594 (дата обращения 15.11.2021).

Поступила 02.02.2022 2.

\section{Информация об авторах}

Eршвв M.C., доктор технических наук, профессор кафедры теоретической электротехники и электрификации нефтяной и газовой промышленности Российского государственного университета нефти и газа (национальный исследовательский университет) имени И.М. Губкина.

Феоктистов E.A., студент Московского физико-технического института (национальный исследовательский университет) 
UDC 62-83:621.313

\title{
ANALYTICAL SOLUTION FOR A SIX-MASS THERMODYNAMIC MODEL OF A TEFC INDUCTION MOTOR
}

\author{
Mikhail S. Ershov ${ }^{1}$, \\ msershov@yandex.ru \\ Eugene A. Feoktistov, \\ Eugene.Feoktistov@yandex.ru \\ 1 National University of Oil and Gas «Gubkin University», \\ 65, bld. 1, Leninsky avenue, Moscow, 119991, Russia. \\ 2 Moscow Institute of Physics and Technology (National Research University), \\ 9, Institutsky lane, Dolgoprudny, 141701, Russia.
}

The relevance of the study is determined by the fact that the thermal operating modes of asynchronous motors largely determine their reliability. Currently, the issues of calculating the steady-state thermal modes of engines are well developed. The issues of practical calculation of transient thermal processes have been worked out less and are limited to numerical modeling of multi-mass schemes or analytical solutions for models with multiple masses. In this paper, a technique is proposed and an analytical solution is obtained for a typical sixmass model of thermal processes of a closed-circuit asynchronous motor - Total Enclosed Fan Cooled. The obtained analytical solutions of a system of inhomogeneous linear differential equations are expressed by a series including a constant component and exponential terms decreasing in time, one of which is dominant, determining the regularization of the machine heating and cooling. The obtained analytical solutions allow us to better understand the patterns of thermal processes of engines when their operating modes change and can serve as the basis for predicting heating and cooling in relay protection systems of asynchronous motors of closed design.

Objective: to develop a methodology for analytical solutions for multi-mass thermodynamic models of electric machines, to obtain an analytical solution for a typical six-mass closed-circuit asynchronous motor system and to analyze the results obtained.

Objects: asynchronous motors in closed design.

Methods: analytical solution of a system of inhomogeneous differential equations.

Results. The authors have developed the method of analytical solutions for multi-mass thermodynamic models of electric machines, obtained the analytical solution for a six-mass system of a closed-circuit asynchronous motor, analyzed the results obtained and established the regularities of the object of study heating regularization.

\section{Key words:}

Inductions motor, Total Enclosed Fan Cooled, thermodynamic model, system of inhomogeneous differential equations, analytical solution, analysis.

\section{REFERENCES}

1. Sipailov G.A., Sannikov D.I., Zhadan V.A. Teplovye, gidravlich eskie $i$ aerodinamicheskie rasschety $v$ electricheskikh mashinakh [Thermal, hydraulic and aerodynamic calculations in electric machines]. Moscow, Vishaya Shkola Publ., 1989. 239 p.

2. Zhadan V.A., Govyazova S.V. Thermal calculation of closedcircuit electric machines with natural cooling and a finned body. Bulletin of the Tomsk Polytechnic University, 2005, vol. 308, no. 7 pp. 174-178. In Rus.

3. Boglietti A., Cavagnino A., Staton D. Thermal analysis of TEFC induction motors. Proceeding IEEE IAS Annual Meeting. Salt Lake City, UK, October 12-16, 2003. pp. 849-856.

4. Boglietti A., Cavagnino A., Staton D. TEFC induction motors thermal models: a parameter sensitivity analysis. IEEE Transactions on Industry Applications, May/June 2005, vol. 41, no. 3, pp. 756-763.

5. German-Galkin S., Sakharov V., Tarnapowicz D. Energy characteristics of asynchronous electric drive. Management Systems in Production Engineering, 2019, vol. 27, no. 1, pp. 51-54. DOI: 10.1515/mspe-2019-0009.

6. Metelkov V.P. On calculating the parameters of a two-mass thermodynamic model of an asynchronous motor. Bulletin of the South Ural State University. Series «Energetika», 2016, vol. 16, no. 1, pp. 58-65. In Rus.

7. Anuchin A.S., Fedorova K.G. A two-mass thermal model of induction motor. Russian Electrical Engineering, 2014, vol. 85, Iss. 2, pp. 83-86.

8. Zyuzev A.M., Kryukov O.V., Metelkov V.P., Mikhalchenko S.G. Assessment of the thermal state of AC electric motors of compressor stations of main gas pipelines. Bulletin of the Tomsk Polytech- nic University. Geo Assets Engineering, 2020, vol. 332, no. 1, pp. 88-96. In Rus.

9. Bugaev G.A., Leontiev E.Yu., Erokhin D.V., Pavlova D.V. Mathematical models of heating and cooling for a microprocessor relay of thermal protection. Electrical Engineering, 2001, no. 2, pp. 51-54. In Rus.

10. Bulychev A.V., Erokhin E.Yu., Pozdeev N.D., Filichev O.A. Thermal model of an asynchronous motor for relay protection purposes. Electrical Engineering, 2011, no. 1, pp. 26-30. In Rus.

11. Graham J., Dixon R., Gregory K., Pearson J. Thermal modelling of an alternator for use in a prediction system. Proc. of 2012 UKACC International Conference on Control (CONTROL 2012). Cardiff, UK, 3-5 September, 2012. pp. 455-460.

12. Venkataraman B., Godsey B., Premerlani W., Shulman E., Thakur M., Midence R. Fundamentals of a motor thermal model and its applications in motor protection. Protective Relay Engineers, 58th Annual Conference. USA, 2005. pp. 11-28.

13. Kuznetsov V., Tryputen M., Tytiuk V., Rozhnenko Z., Levchenko S., Kuznetsov V. Modeling of thermal process in the energy system «electrical network - asynchronous motor». E3S Web of Conferences ICSF, 2021, pp. 1-6. Available at: https://doi.org/ 10.1051/e3sconf/202128005003 (accessed 15 November 2021).

14. Roland P. MATLAB for electrical and computer engineering students and professional with Simulink. Edison, SciTech Publ., NJ. University of Illinois at Chicago, 2013. 662 p.

15. Kondratiev G.M. Regularny theplovoy rezhim [Regular thermal regime]. Moscow, State Publ. house of Technical and Theoretical Literature, 1954. $408 \mathrm{p}$.

16. Hanappier N., Charkaluk E., Triantafyllidis N. A coupled electromagnetic-thermomechanical approach for the modeling of electric 
motors. Journal of the Mechanics and Physics of Solids, 2021, vol. 149, pp. 1-31. DOI: 10.1016/j.jmps.2021.104315.

17. Zocholl S. Optimizing motor thermal models. Proceeding. Industrial \& Commercial Power Systems Technical Conference, ICPS 2007. Edmonton, Alberta, Canada, 6-11 May 2007. pp. 33-41

18. Borodin M.Yu., Borodin E.M., Metelkov V.P. Application of integral equations for analysis of electrodynamic and thermal processes in electric drive. $2^{\text {nd }}$ International Conference on Industrial Engineering, Applications and Manufacturing (ICIEAM 2016). Chelyabinsk, Russia, 19-20 May 2016. pp. 1-4.

19. Khomchenko D.N., Kovalev G.V. Investigation of the effect of increased current density in brushes on heating an electric locomotive traction motor. Vestnik RGUPS, 2019, no. 1, pp. 46-52. In Rus.
20. Staton D., Boglietti A., Cavagnino A. Solving the more difficult aspects of electric motor thermal analysis in small and medium size industrial induction motors. IEEE Transaction on Energy Conversion, 2005, vol. 20, no. 3, pp. 620-628.

21. Korenciak D., Sebok M., Gutten M. Thermal measurement and its application for diagnostics of distribution oil transformers. Energetika Proceedings of CIS higher education institutions and power engineering associations, 2019, vol. 62, no. 6, pp. 583-594. In Rus. Available at: https://doi.org/10.21122/1029-7448-2019-62-6-583594 (accessed 15 November 2021).

Received: 2 February 2022.

\section{Information about the authors}

Mikhail S. Ershov, Dr. Sc., professor, National University of Oil and Gas «Gubkin University».

Eugene A. Feoktistov, student, Moscow Institute of Physics and Technology (National Research University). 